\title{
On degradation of Grover's search under collective phase flips in queries to the oracle
}

\author{
Alexey E. Rastegin \\ Department of Theoretical Physics, Irkutsk State University, \\ Russia, e-mail: alexrastegin@mail.ru
}

\begin{abstract}
We address the case, when querying to the oracle in Grover's algorithm is exposed to noise including phase distortions. The oracle-box wires can be altered by an opposite party that tries to prevent correct receiving data from the oracle. This situation reflects an experienced truth that any access to prophetic knowledge cannot be common and direct. To study the problem, we introduce a simple model of collective phase distortions on the base of phase damping channel. In the model used, the success probability is not altered via the oracle-box wires per se. Phase distortions of the considered type can hardly be detected via any one-time query to the oracle. However, the success probability is significantly changed, when such errors are introduced as an intermediate step into the Grover iteration. We investigate the success probability with respect to variations the parameter that characterizes the amount of phase errors. It turns out that the success probability is decreased significantly even if the error amount is not very high. Moreover, this probability quickly reduces to the value of one half, which corresponds to the completely mixed state. We also study trade-off relations between quantum coherence and the success probability in the presence of noise of the considered type.
\end{abstract}

Keywords: Grover's algorithm, phase noise, relative entropy of coherence

\section{INTRODUCTION}

In last decades, quantum effects have found a new field of applications for information processing [1]. Famous Shor's discovery [2] had lead to numerous quantum algorithms for algebraic problems [3 [5]. Grover's search algorithm [6-8] is another fundamental result. Inspired amplitude amplification technique is widely used as one of primary tools in building quantum algorithms [9]. The algorithms of Shor and Grover may be related more closely than it seems initially [10]. Studying of quantum algorithms is a part of recent efforts to realize emerging technologies in quantum information processing. The Grover algorithm is optimal for search by means of queries to the oracle [11, 12]. The user invoke the oracle to process any item, whereas the database itself is not represented explicitly. The original formulation has been modified with particular blocks of more general kind. In addition, amplification process may start with an arbitrary initial distribution of the amplitudes. Generalized versions of Grover's algorithm were thoroughly analyzed in [13 16].

Algorithms of amplitude amplification are related to the case, when users employ an access to the so-called oracle. The oracle denotes some black box, which is able to calculate values of the desired Boolean function. Users query the box by putting concrete values of the argument. In reality, an access to the oracle may be impeded and unreliable. Due to a wide applicability of amplitude amplification technique, this problem deserves detailed investigations. The oracle-box wires are inevitably exposed to noise, even if its amount is low. In addition, the wires could be affected due to activity of an opposite party. There are many possible scenarios, in which the above questions could be examined. In this work, we address one of such scenarios. Despite of its simplicity, we disclose some unexpected corollaries of collective phase flips in the oracle-box wires. In the model considered, queries to the oracle are exposed to phase flips described similarly to the phase damping of a qubit. We also discuss the relative entropy of coherence from the viewpoint of its trade-off with the success probability.

The paper is organized as follows. The preliminary material is given in Sect. III In Sect. III] we introduce the model of collective flips that occurs in the oracle-box wires. The used model leads to the recursion equation in terms of the effective Bloch vector. From the viewpoint of noise amount, two certain cases should be distinguished. In Sect. IV] we examine changes of the success probability after repeated Grover's iterations under collective phase flips. It turns out that the Grover search algorithm is very sensitive to distortions of the considered type. Using the relative entropy of coherence, we further study trade-off relations with the success probability. In Sect. $\nabla$ we conclude the paper with a summary of results. Appendix $\mathrm{A}$ is devoted to solutions of the derived recursion equation.

\section{PRELIMINARIES}

In this section, the required material will be recalled. We begin with a lot of linear algebra. Some aspects of the Grover search algorithm, quantum noise and coherence measures will also be presented. For a rectangular $m \times n$ matrix $\mathbf{Z}$, its singular values $s_{j}(\mathbf{Z})$ are defined as square root of the eigenvalues of positive semidefinite matrix $Z^{\dagger} Z$ [17]. The number of non-zero singular values is equal to $\operatorname{rank}(\mathbf{Z}) \leq \min \{m, n\}$. The matrices $\mathbf{Z}^{\dagger} \mathbf{Z}$ and $\mathbf{Z Z}^{\dagger}$ have the same non-zero eigenvalues. For $q \in[1 ; \infty]$, the 
Schatten $q$-norm is defined as $[17$

$$
\|\mathrm{Z}\|_{q}:=\left(\sum_{j=1}^{\operatorname{rank}(\mathrm{Z})} s_{j}(\mathrm{Z})^{q}\right)^{1 / q} .
$$

In the following, we will use the Frobenius norm $\|Z\|_{2}:=$ $\sqrt{\operatorname{tr}\left(\mathbf{Z}^{\dagger} \mathbf{Z}\right)}$ and the spectral norm $\|Z\|_{\infty}:=\max s_{j}(\mathbf{Z})$. One of important properties of the Schatten norms is expressed by the inequality (see, e.g., formula (1.175) in [17])

$$
\|\mathrm{XYZ}\|_{q} \leq\|\mathrm{X}\|_{\infty}\|\mathrm{Y}\|_{q}\|\mathrm{Z}\|_{\infty}
$$

Let us recall the original formulation of Grover's search algorithm. The search space contains $N=2^{n}$ items denoted by binary $n$-string $x=\left(x_{1} \cdots x_{n}\right)$ with $x_{j} \in\{0,1\}$ so that $x \in\{0,1, \ldots, N-1\}$. The problem is to find one of marked items that form some set $\mathcal{M}$. By $\mathcal{M}^{\mathrm{C}}$, we mean the complement of this set. Without loss of generality, we can assume $1 \leq|\mathcal{M}| \leq N / 2$.

Checking items, the algorithm appeals to the so-called "oracle". For the each given $x$, the oracle returns the value of Boolean function $x \mapsto F(x)$ such that $F(x)=1$ for $x \in \mathcal{M}$ and $F(x)=0$ for $x \in \mathcal{M}^{\mathrm{C}}$. Initially, the algorithm initializes the $n$-qubit register to $|0\rangle$. Applying the Hadamard transform, one gets the distribution with equal amplitudes, namely

$$
\mathrm{H}|0\rangle=\frac{1}{\sqrt{N}} \sum_{x=0}^{N-1}|x\rangle .
$$

Such superpositions are used to realize the quantum parallelism [18]. Furthermore, we repeat the Grover iteration involving two steps. The first step with querying the oracle can be represented by the rotation operator

$$
\mathrm{J}=\sum_{x=0}^{N-1}(-1)^{F(x)}|x\rangle\langle x| .
$$

By (4), amplitudes of marked states are all multiplied by the phase factor $\exp (i \pi)=-1$. This step has been generalized to other values of the phase [13, 15]. Since we shall focus on an influence of noise, such generalizations are not considered in the following. The second step of the Grover iteration realizes the inversion about mean [19]. It is described by the operator

$$
\mathrm{K}=2 \mathrm{H}|0\rangle\langle 0| \mathrm{H}-\mathbb{1}_{N},
$$

where $\mathbb{1}_{N}$ is the identity operator of the corresponding size. We can sometimes replace (5) with a more general block. Such algorithms were analyzed in [13, 15]. Thus, the standard Grover iteration is written as

$$
\mathrm{G}=\mathrm{KJ} .
$$

In the standard formulation, the initial distribution of amplitudes is taken in the form (3). Then the evolution of amplitudes can be described within the two-dimensional picture. Let us define the normalized superpositions of unmarked and marked states,

$$
\begin{aligned}
|w\rangle & :=\frac{1}{\sqrt{N-M}} \sum_{x \in \mathcal{M}^{\mathrm{c}}}|x\rangle . \\
|m\rangle & :=\frac{1}{\sqrt{M}} \sum_{x \in \mathcal{M}}|x\rangle,
\end{aligned}
$$

We will also use the parameter $\theta \in(0 ; \pi / 2)$ such that $\cos \theta=1-2 M / N$, whence

$$
\sin ^{2} \theta / 2=\frac{M}{N}, \quad \cos ^{2} \theta / 2=1-\frac{M}{N} .
$$

Writing operators as matrices in the basis $\{|w\rangle,|m\rangle\}$, we have $\mathrm{J}=\operatorname{diag}(+1,-1)=\boldsymbol{\sigma}_{z}$ and

$$
\mathrm{K}=\left(\begin{array}{cc}
\cos \theta & \sin \theta \\
\sin \theta & -\cos \theta
\end{array}\right) .
$$

Thus, the operator (6) is simply represented as [19]

$$
\mathrm{G}=\left(\begin{array}{cc}
\cos \theta & -\sin \theta \\
\sin \theta & \cos \theta
\end{array}\right)
$$

Thus, each Grover iteration rotates the register state by $\theta$ towards the superposition $|m\rangle$. We wish to address the case when querying to the oracle is exposed to noise. To obtain explicit results, we have to restrict a consideration on sufficiently simple models of errors.

Entanglement is one of key resources in quantum computations. Due to findings of the papers [20, 21], quantum speed-up without entanglement is hardly possible. To analyze the nature of quantum algorithms, we should think about correlations in the context of few prescribed bases. This question is related to changes of quantum coherence of the register during computational processes. The framework for studies of coherence as purely quantum feature was developed in recent years [22, 23]. Concerning amplitude amplification, trade-off relations between quantum coherence and the success probability is one of important questions. We shall address this question in the case, when queries to the oracle are exposed to phase flips of the considered type. To do so, the relative entropy of coherence will be utilized.

In general, various approaches to measure quantum correlations are discussed in [23 26]. The authors of [22] developed a list of axioms that should be satisfied by any proper quantifier of coherence. As a rule, each candidate to quantify the amount of coherence is associated with some distinguishability measure. Let us take the set $\mathcal{I}$ of all diagonal density matrices, namely

$$
\boldsymbol{\delta}=\sum_{x=0}^{N-1} b(x)|x\rangle\langle x|, \quad \sum_{x=0}^{N-1} b(x)=1 .
$$

We asks how far the given state is from those states that are completely incoherent in the computational basis. Using the quantum relative entropy as a measure of 
distinguishability leads to the relative entropy of coherence. The quantum relative entropy of $\boldsymbol{\rho}$ with respect to $\boldsymbol{\omega}$ is defined as $[17,27]$

$$
D_{1}(\boldsymbol{\rho} \| \boldsymbol{\omega}):= \begin{cases}\operatorname{tr}(\boldsymbol{\rho} \ln \boldsymbol{\rho}-\boldsymbol{\rho} \ln \boldsymbol{\omega}), & \text { if } \operatorname{ran}(\boldsymbol{\rho}) \subseteq \operatorname{ran}(\boldsymbol{\omega}), \\ +\infty, & \text { otherwise }\end{cases}
$$

By $\operatorname{ran}(\boldsymbol{\rho})$, we mean here the range of $\boldsymbol{\rho}$. On the base of (13), the corresponding coherence measure is introduced as 22]

$$
C_{1}(\boldsymbol{\rho}):=\min _{\boldsymbol{\delta} \in \mathcal{I}} D_{1}(\boldsymbol{\rho} \| \boldsymbol{\delta}) .
$$

The minimization leads to the expression [22]

$$
C_{1}(\boldsymbol{\rho})=S_{1}\left(\boldsymbol{\rho}_{\mathrm{diag}}\right)-S_{1}(\boldsymbol{\rho}),
$$

where $S_{1}(\boldsymbol{\rho})=-\operatorname{tr}(\boldsymbol{\rho} \ln \boldsymbol{\rho})$ is the von Neumann entropy of $\rho$, and

$$
\boldsymbol{\rho}_{\text {diag }}:=\sum_{x=0}^{N-1} p(x)|x\rangle\langle x|, \quad p(x)=\langle x|\boldsymbol{\rho}| x\rangle .
$$

The entropy $S\left(\boldsymbol{\rho}_{\text {diag }}\right)$ is equal to the Shannon entropy calculated with the probabilities $p(x)$. Basic properties of (14) are considered in [22, 23]. Generalized entropic functions have found use in quantum information theory. It is for this reason that we designate the above quantities by the subscript 1 . Coherence quantifiers induced by quantum divergences of the Tsallis type were addressed in 28]. It was shown that such quantifiers do not allow a simple form similar to (15). Coherence monotones based on Rényi divergences were considered in 29 31]. Other candidates to quantify the amount of coherence were examined in [32, 33]. The geometric coherence is an interesting quantifier of different character 23]. Twosided estimates on the geometric coherence were obtained in [34].

Complementarity relations for quantum coherence can be formulated in several ways [35 39]. Duality relations between the coherence and path information were examined in 40 42]. From the viewpoint of quantum computations, the concept of quantum coherence was studied in [43 45]. In particular, the authors of [44] reported on coherence depletion in the original Grover algorithm. In the paper [46], we have studied relations between coherence and the success probability in generalized amplitude amplification. Some of these results will be used in studies of Grover's search in the presence of phase flips.

\section{COLLECTIVE FLIPS INTRODUCED BY PHASE DAMPING}

There are infinitely many scenarios of an interaction with environment. We will consider a model with phase damping. Such processes describe the loss of quantum information without loss of energy [19]. It gives a ground for understanding physical effects in quantum systems similar to the Schrödinger cat-atom system. Let us focus on those density matrices that are effectively twodimensional with respect to the basis $\{|w\rangle,|m\rangle\}$. In other words, they can be represented via usual Bloch vector $\mathbf{r}=\left(r_{x}, r_{y}, r_{z}\right)$, namely

$$
\boldsymbol{\rho}=\frac{1}{2}\left(\begin{array}{cc}
1+r_{z} & r_{x}-\mathbf{i} r_{y} \\
r_{x}+\mathrm{i} r_{y} & 1-r_{z}
\end{array}\right) .
$$

With positive parameter $\eta \leq 1$, we introduce the following Kraus operators,

$$
\mathrm{E}_{0}:=\left(\begin{array}{cc}
1 & 0 \\
0 & \sqrt{\eta}
\end{array}\right), \quad \mathrm{E}_{1}:=\left(\begin{array}{cc}
0 & 0 \\
0 & \sqrt{1-\eta}
\end{array}\right) .
$$

These operators prescribe the action of phase damping $\Phi_{\mathrm{E}}$ on density matrices of the above type. It is well known that this action reads as [19]

$$
\left(r_{x}, r_{y}, r_{z}\right) \stackrel{\Phi_{\mathrm{E}}}{\longmapsto}\left(\sqrt{\eta} r_{x}, \sqrt{\eta} r_{y}, r_{z}\right) .
$$

Initializing gives the density matrix $\boldsymbol{\rho}(0)=\mathrm{H}|0\rangle\langle 0| \mathrm{H}$ with the Bloch vector $\mathbf{r}(0)=(\sin \theta, 0, \cos \theta)$. After $t$ iterations, the success probability is written as

$$
P_{\text {suc }}(t)=\langle m|\boldsymbol{\rho}(t)| m\rangle=\frac{1-r_{z}(t)}{2} .
$$

It must be stressed that the channel $\Phi_{\mathrm{E}}$ itself cannot alter the probability of the success. We immediately see this fact from (18). Nevertheless, the probability of the success may be changed in the case, when this channels acts as an intermediate point in amplitude amplification process.

We will investigate the following scheme. Each iteration transforms density matrices of the register according to the formula

$$
\boldsymbol{\rho}(t) \mapsto \boldsymbol{\rho}(t+1)=\Upsilon_{\mathrm{K}} \circ \Phi_{\mathrm{E}} \circ \Upsilon_{\mathrm{J}} \circ \Phi_{\mathrm{E}}(\boldsymbol{\rho}(t)),
$$

where the two unitary channels are written as

$$
\Upsilon_{J}(\varrho)=\mathrm{J} \varrho \mathrm{J}^{\dagger}, \quad \Upsilon_{\mathrm{K}}(\varrho)=\mathrm{K} \varrho \mathrm{K}^{\dagger} .
$$

For $\eta=1$, the map $\Phi_{\mathrm{E}}$ reads as identical, so that the right-hand side of (20) takes the form

$$
\boldsymbol{\rho}(t+1)=\Upsilon_{\mathbf{K}} \circ \Upsilon_{\mathcal{J}}(\boldsymbol{\rho}(t))=\mathrm{G} \boldsymbol{\rho}(t) \mathrm{G}^{\dagger} .
$$

If the initialized state is pure, then the register will remain to be in pure states under the action of the map (22). It is not the case for the altered map (20).

The above model deals with collective phase distortions that are simply expressed in the computational basis. It allows us to formulate results in a closed analytic form. Under some circumstances, the considered picture concerns the case when phase flips occur in a single qubit solely. The phase flip channel has Kraus operators $\sqrt{\alpha} \mathbb{1}_{2}$ and $\sqrt{1-\alpha} \boldsymbol{\sigma}_{z}$ [19]. This channel is actually equivalent to the phase damping channel, whenever

$$
2 \alpha=1+\sqrt{\eta} .
$$


If only one of qubits is affected, then we can split the total Hilbert space into two subspaces $\mathcal{H}_{0}$ and $\mathcal{H}_{1}$. These subspaces are spanned by canonical states that have in their binary notation either 0 or 1 in the position of the noised qubit, respectively. Then any density matrix $\rho$ can be written as a block $2 \times 2$-matrix $\left[\left[\boldsymbol{\rho}_{i j}\right]\right]$. Due to phase flips in the noised qubit, we have $\boldsymbol{\rho}_{i j} \mapsto \sqrt{\eta} \boldsymbol{\rho}_{i j}$ for $i \neq j$. The diagonal submatrices $\boldsymbol{\rho}_{00}$ and $\boldsymbol{\rho}_{11}$ are not changed. Suppose that errors occur in each query to the oracle. In general, a complete analysis is complicated [47]. However, the situation can sometimes be reduced to our model. When one of subspaces consists of only marked states, we really deal with the channel $\Phi_{E}$ due to (18). Our scenario does not mean that phase distortions act in all qubits simultaneously. Dephasing noise may be treated as a result of the existence of "damaged" vertices in quantum-walk search [47]. Due to an interferometric picture of quantum walks [48], one can therefore give an example of phase errors in realistic systems.

Interpreting (20) as a matrix relation, we will solve it via the diagonalization. This approach is somehow similar to the treatment of [15]. On the other hand, we deal with the recursion equation for components of the actual Bloch vector. The authors of [15] used the recursion equation for components of the wave function. In our case, only two of components of the Bloch vector are non-zero. So, we will further treat $\mathbf{r}(t)$ as a column with two entries, namely $r_{x}(t)$ and $r_{z}(t)$. In terms of the Bloch vector components, the action of operation $\Phi_{\mathrm{E}}$ is represented by the matrix $\operatorname{diag}(\sqrt{\eta},+1)$. We also have

$$
\begin{aligned}
2 \mathrm{~J} \rho \boldsymbol{J}^{\dagger} & =\boldsymbol{\sigma}_{z}\left(\mathbb{1}_{2}+r_{x} \boldsymbol{\sigma}_{x}+r_{z} \boldsymbol{\sigma}_{z}\right) \boldsymbol{\sigma}_{z} \\
& =\mathbb{1}_{2}-r_{x} \boldsymbol{\sigma}_{x}+r_{z} \boldsymbol{\sigma}_{z},
\end{aligned}
$$

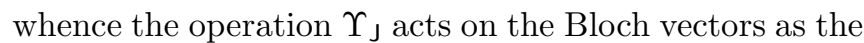
matrix $\operatorname{diag}(-1,+1)$. Using (10), we obtain $\mathrm{K}_{2} \mathrm{~K}^{\dagger}=\mathbb{1}_{2}$,

$$
\begin{aligned}
& \mathrm{K} \boldsymbol{\sigma}_{x} \mathrm{~K}^{\dagger}=-\cos 2 \theta \boldsymbol{\sigma}_{x}+\sin 2 \theta \boldsymbol{\sigma}_{z}, \\
& \mathrm{~K} \boldsymbol{\sigma}_{z} \mathrm{~K}^{\dagger}=\sin 2 \theta \boldsymbol{\sigma}_{x}+\cos 2 \theta \boldsymbol{\sigma}_{z},
\end{aligned}
$$

so that $r_{x} \mapsto-\cos 2 \theta r_{x}+\sin 2 \theta r_{z}$ and $r_{z} \mapsto \sin 2 \theta r_{x}+$ $\cos 2 \theta r_{z}$ due to the operation $\Upsilon_{\mathrm{K}}$. Hence, this operation acts on the Bloch vectors as the matrix

$$
\left(\begin{array}{cc}
-\cos 2 \theta & \sin 2 \theta \\
\sin 2 \theta & \cos 2 \theta
\end{array}\right)
$$

Finally, the recursion equation is written in terms of the effective Bloch vector as

$$
\mathbf{r}(t+1)=\operatorname{Lr}(t),
$$

where the matrix $L$ reads as

$$
\mathbf{L}=\left(\begin{array}{cc}
\eta \cos 2 \theta & \sin 2 \theta \\
-\eta \sin 2 \theta & \cos 2 \theta
\end{array}\right) \text {. }
$$

Thus, we obtain the equation $\mathbf{r}(t)=\mathrm{L}^{t} \mathbf{r}(0)$ solved explicitly in Appendix $\mathrm{A}$. The singular values of $L$ are equal to
1 and $\eta$, whence $\|\mathrm{L}\|_{\infty}=1$. Combining the latter with (2) implies that the 2-norm of the Bloch vector cannot increase, i.e., $\|\mathbf{r}(t)\|_{2} \leq\|\mathbf{r}(0)\|_{2}$.

It will be convenient to put three positive parameters, viz.

$$
\begin{aligned}
A_{ \pm}: & :=\frac{1 \pm \eta}{2} \cos 2 \theta, \\
B & := \begin{cases}\sqrt{\eta-A_{+}^{2}}, & \text { if } \eta>A_{+}^{2}, \\
0, & \text { if } \eta=A_{+}^{2}, \\
\sqrt{A_{+}^{2}-\eta}, & \text { if } \eta<A_{+}^{2} .\end{cases}
\end{aligned}
$$

The characteristic equation is written as

$$
\lambda^{2}-2 A_{+} \lambda+\eta=0 .
$$

We will further assume that $A_{+}^{2}-\eta \neq 0$. Then the eigenvalues $\lambda_{+}$and $\lambda_{-}$differ so that the matrix $L$ is certainly diagonalizable. The following two cases should be mentioned: (i) $\eta>A_{+}^{2}$; (ii) $\eta<A_{+}^{2}$. In Appendix [ $\mathrm{A}$ we examine them separately. In the first case, one has

$$
\begin{aligned}
r_{z}(t)=\frac{\eta^{t / 2}}{B} & (-\eta \sin \varphi t \sin 2 \theta \sin \theta \\
& \left.+\left(B \cos \varphi t+A_{-} \sin \varphi t\right) \cos \theta\right), \\
P_{\mathrm{suc}}(t)=\frac{1}{2 B} & \left(B+\eta^{1+t / 2} \sin \varphi t \sin 2 \theta \sin \theta\right. \\
& \left.-\eta^{t / 2}\left(B \cos \varphi t+A_{-} \sin \varphi t\right) \cos \theta\right) .
\end{aligned}
$$

In the second case, one gets

$$
\begin{aligned}
r_{z}(t)=\frac{\eta^{t / 2}}{B} & (-\eta \sinh \phi t \sin 2 \theta \sin \theta \\
& \left.+\left(B \cosh \phi t+A_{-} \sinh \phi t\right) \cos \theta\right), \\
P_{\mathrm{suc}}(t)=\frac{1}{2 B} & \left(B+\eta^{1+t / 2} \sinh \phi t \sin 2 \theta \sin \theta\right. \\
& \left.-\eta^{t / 2}\left(B \cosh \phi t+A_{-} \sinh \phi t\right) \cos \theta\right) .
\end{aligned}
$$

\section{ON DYNAMICS OF THE SUCCESS PROBABILITY AND QUANTUM COHERENCE}

In this section, we will use the solutions (32) and (34) for studying a vulnerability of Grover's search with respect to phase flips in the oracle-box wires. In principle, they may be inspired by an opposite party that tries to prevent correct querying to the oracle. To input collective phase flips, the opponent should be aware of details of the Boolean function $x \mapsto F(x)$.

To study significance of collective phase flips, we visualize $P_{\text {suc }}(t)$ versus $t$ for several values of the parameter $\eta$. We begin with the case (i), when $\eta>A_{+}^{2}$. As was mentioned above, this case includes the standard situation $\eta=1$. In Fig. 1 we take $N=64, M=1$, and 


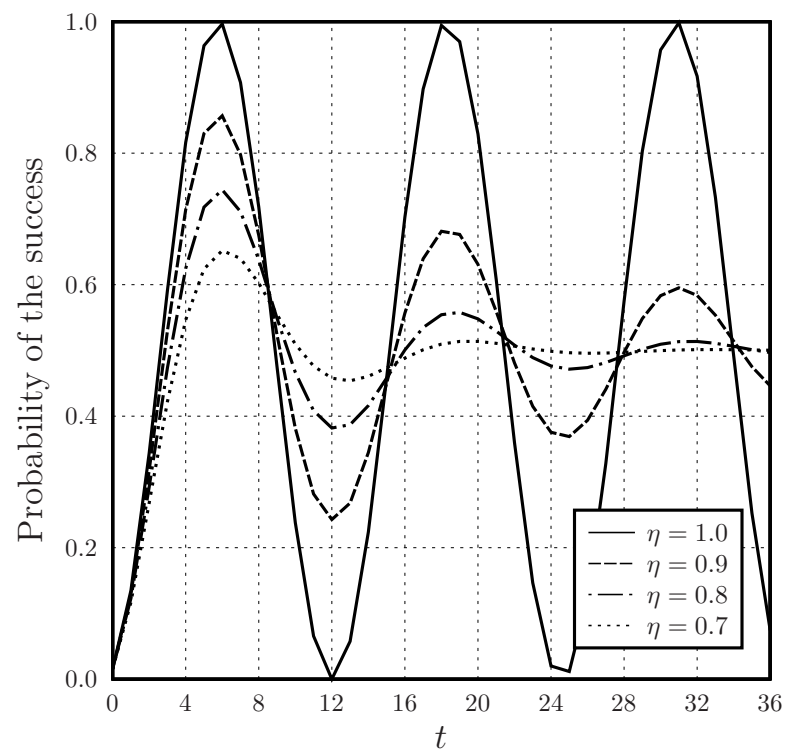

FIG. 1: In the case (i), $P_{\text {suc }}(t)$ is shown for $N=64, M=1$, and the four values of $\eta$.

show the lines for the four values of $\eta$. Although $t$ takes integer values, one draws lines as continuous for the sake of visibility. Even if the amount of errors is low, values of the success probability are reduced essentially. Without distortions, when $\eta=1$, the dependence of $P_{\text {suc }}(t)$ on $t$ is almost periodic. Noticeable decreasing of $P_{\text {suc }}(t)$ due to $\eta<1$ is observed even in the first cycle of amplitude amplification. After several cycles, the curve $P_{\text {suc }}(t)$ asymptotically degenerates to the constant equal to $1 / 2$. The same result was later reported for a particular case of decoupling noise in [47].

For the completeness, we also show an example of $P_{\text {suc }}(t)$ for the case (ii), when $\eta<A_{+}^{2}$. In Fig. 2, we set $N=64, M=1$, and show the lines for the four low values of $\eta$. Here, the dependence $P_{\text {suc }}(t)$ is of a completely different character. Instead of decaying cycles with some peaks, we observe that the lines smoothly and quickly saturate the constant equal to $1 / 2$. This behavior cannot be treated as amplitude amplification of any kind. When the oracle-box wires are exposed to the channel $\Upsilon_{K}$ with such low values $\eta$, legitimate users are rather able to detect this fact. So, we further return to the case (i) and address trade-off relations between quantum coherence and the success probability.

The relative entropy of coherence satisfies [46]

$$
\begin{aligned}
& h_{1}\left(P_{\mathrm{suc}}\right) \leq C_{1}(\boldsymbol{\rho})+S_{1}(\boldsymbol{\rho}) \\
& \leq P_{\mathrm{suc}} \ln \left(\frac{M}{P_{\mathrm{suc}}}\right)+\left(1-P_{\mathrm{suc}}\right) \ln \left(\frac{N-M}{1-P_{\mathrm{suc}}}\right),
\end{aligned}
$$

where $h_{1}\left(P_{\text {suc }}\right)$ is the binary Shannon entropy. Calculating $C_{1}(\boldsymbol{\rho}(t))$, we examine it from the viewpoint of (35).

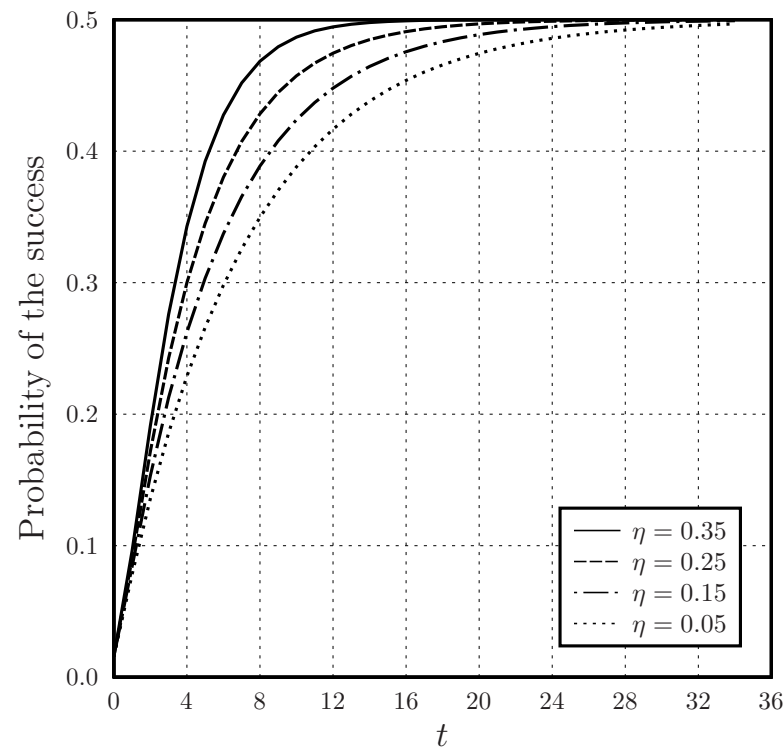

FIG. 2: In the case (ii), $P_{\text {suc }}(t)$ is shown for $N=64, M=1$, and the four values of $\eta$.

To do so, we recall the complete form of $\boldsymbol{\rho}(t)$, namely

$$
\begin{aligned}
\boldsymbol{\rho}(t)= & \frac{1+r_{z}(t)}{2}|w\rangle\langle w|+\frac{r_{x}(t)}{2}(|w\rangle\langle m|+| m\rangle\langle w|) \\
& +\frac{1-r_{z}(t)}{2}|m\rangle\langle m| .
\end{aligned}
$$

In the computational basis, the diagonal part of $\boldsymbol{\rho}(t)$ can be written as the diagonal matrix, which has the value $\left(1-P_{\text {suc }}(t)\right) /(N-M)$ with multiplicity $N-M$ and the value $P_{\text {suc }}(t) / M$ with multiplicity $M$. For $t>0$, the non-zero eigenvalues of $\boldsymbol{\rho}(t)$ are obtained as

$$
\frac{1 \pm\|\mathbf{r}(t)\|_{2}}{2}, \quad\|\mathbf{r}(t)\|_{2}=\sqrt{r_{x}(t)^{2}+r_{z}(t)^{2}}<1 .
$$

Of course, with the initial distribution (3) we have $\|\mathbf{r}(0)\|_{2}=1$. Due to (A10), we have $\|\mathbf{r}(t)\|_{2} \propto \eta^{t / 2}$. In the considered case of amplitude amplification, we have the equality

$$
\begin{aligned}
C_{1}(\boldsymbol{\rho}(t))= & P_{\mathrm{suc}}(t) \ln \left(\frac{M}{P_{\mathrm{suc}}(t)}\right) \\
& +\left(1-P_{\mathrm{suc}}(t)\right) \ln \left(\frac{N-M}{1-P_{\mathrm{suc}}(t)}\right)-S_{1}(\boldsymbol{\rho}(t)) .
\end{aligned}
$$

That is, the upper bound of the right-hand side of (35) is saturated here. For the given $P_{\text {suc }}$, this upper bound approves the maximal possible value of $C_{1}(\boldsymbol{\rho})$. We see that trade-offs between $C_{1}(\boldsymbol{\rho}(t))$ and $P_{\text {suc }}(t)$ follow the mentioned line. Let us exemplify a dependence of the relative entropy of coherence on the step number $t$. In Fig. 3. we take $N=64, M=1$, and show $C_{1}(\boldsymbol{\rho}(t))$ for the four values of $\eta$. Except for the value $\eta=1.0$, the curves asymptotically lie on the constant line. This constant is generally written as $(1 / 2) \ln \left(M N-M^{2}\right)$. Substituting 


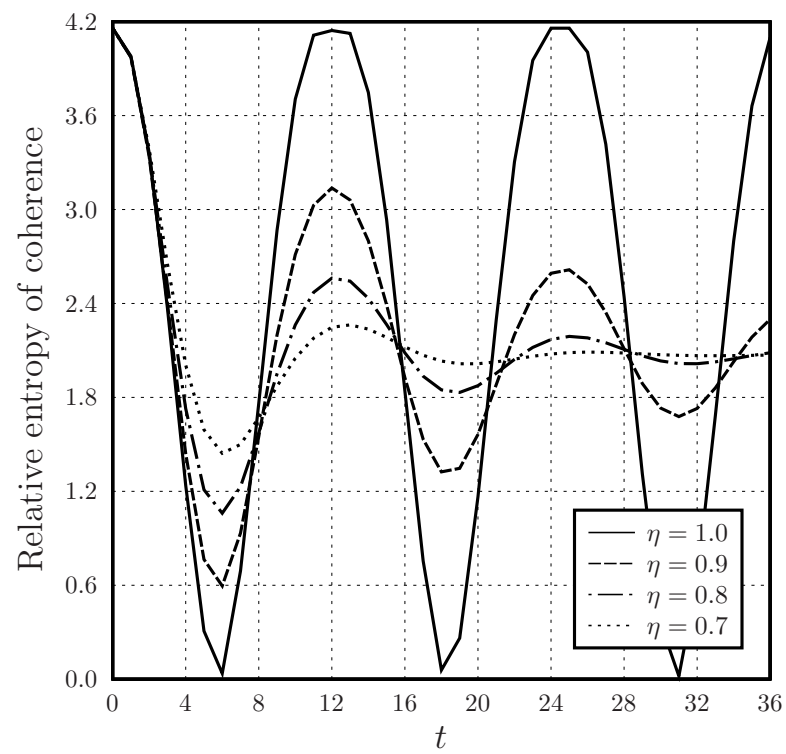

FIG. 3: In the case (i), $C_{1}(\boldsymbol{\rho}(t))$ is shown for $N=64, M=1$, and the four values of $\eta$.

$N=64$ and $M=1$, we have the value $\ln 63 / 2 \approx 2.072$, which is also seen in Fig. 3. For the value $\eta=1$, we may compare the two curves in Figs. 1 and 3 . One observes that peaks of $P_{\text {suc }}(t)$ correspond to valleys of $C_{1}(\boldsymbol{\rho}(t))$, and vice versa. In detail, this property was discussed in [44]. We also observe that the curves for $\eta<1$ reveal a similar behavior but now with decaying. Even if the amount of phase noise is low, oscillations of of the relative entropy are reduced sufficiently quickly. These results additionally maintain the conclusions previously reported in [46]. Namely, even tight trade-off relation between coherence and the success probability does not imply a high quality of amplitude amplification. This question deserves further investigations.

\section{CONCLUSIONS}

We have examined the case, when queries to the oracle in Grover's search algorithm are exposed to phase distortions of the specific type. Another possibility is that the oracle-box wires are altered due to intrusion of an opposite party. The used model of collective phase flips is similar to the phase damping channel. Despite of its simplicity, this model allows us to observe some genuine features of amplitude amplification processes. We have concluded that Grover's search algorithm is actually sensitive to collective phase flips occurring in the oracle-box wires. This feature also provides an opposite party with chances to prevent proper queries of legitimate users to the oracle. At the same time, phase flips are such that the value of the success probability is not changed during transfer via these wires. Even if the user has been ensured with testing states, he is hardly able to detect such distortions by means of one-time queries to the oracle.
We also investigated trade-off relation between coherence and the success probability under noise of the considered type. Our findings have further supported the conclusions obtained previously.

\section{Appendix A: Solutions of the recursion equation}

To solve (26), we calculate the eigenvalues and the corresponding eigenvectors. We begin with the case (i), when $\eta>A_{+}^{2}$. By calculations, one has

$$
\cos 2 \theta=1-\frac{8 M}{N}+\frac{8 M^{2}}{N^{2}} .
$$

The most interesting case occurs, when $M \ll N$ and the term $\cos 2 \theta$ is sufficiently close to 1 . The condition of the case (i),

$$
\frac{2 \sqrt{\eta}}{1+\eta}>\cos 2 \theta
$$

will be fulfilled for $\eta_{\min }<\eta<1$ with

$$
\sqrt{\eta_{\min }}=\frac{1-\sin 2 \theta}{\cos 2 \theta} \text {. }
$$

The value $\eta_{\min }$ does not approach 1 with necessity, whence the above range may be wide enough. Say, for $M=1$ and $N=64$ we get $\cos 2 \theta \approx 0.877$ and $\eta_{\min } \approx 0.351$. Due to $B^{2}=\eta-A_{+}^{2}$, the eigenvalues are written in the form

$$
\lambda_{ \pm}=A_{+} \pm \mathrm{i} B
$$

Using $A_{+}^{2}+B^{2}=\eta$, put positive angle $\varphi$ such that

$$
\begin{aligned}
\frac{A_{+}}{\sqrt{\eta}} & =\cos \varphi, \quad \frac{B}{\sqrt{\eta}}=\sin \varphi, \\
\varphi & =\arctan \left(\frac{B}{A_{+}}\right) .
\end{aligned}
$$

The eigenvalues are rewritten as $\lambda_{ \pm}=\sqrt{\eta} \exp ( \pm i \varphi)$. Calculating the corresponding eigenvectors, we further obtain

$$
X^{-1} \mathrm{LX}=\mathrm{D},
$$

where $\mathrm{D}=\operatorname{diag}\left(\lambda_{+}, \lambda_{-}\right)$and

$$
\begin{aligned}
& \mathbf{X}=\left(\begin{array}{cc}
\sin 2 \theta & \sin 2 \theta \\
A_{-}+\mathrm{i} B & A_{-}-\mathrm{i} B
\end{array}\right), \\
& \mathrm{X}^{-1}=\frac{1}{2 \mathrm{i} B \sin 2 \theta}\left(\begin{array}{lc}
\mathrm{i} B-A_{-} & \sin 2 \theta \\
\mathrm{i} B+A_{-} & -\sin 2 \theta
\end{array}\right) \text {. }
\end{aligned}
$$

Calculations of the matrix $\mathrm{L}^{t}=\mathrm{XD}^{t} \mathrm{X}^{-1}$ finally give

$$
\frac{\eta^{t / 2}}{B}\left(\begin{array}{cc}
B \cos \varphi t-A_{-} \sin \varphi t & \sin \varphi t \sin 2 \theta \\
-\eta \sin \varphi t \sin 2 \theta & B \cos \varphi t+A_{-} \sin \varphi t
\end{array}\right) .
$$


Due to $r_{x}(0)=\sin \theta$ and $r_{z}(0)=\cos \theta$, the above formulas result in (31) and (32). For $\eta=1$, when the oracle-we have $A_{+}=\cos 2 \theta, A_{-}=0, B=\sin 2 \theta$, and $\varphi=2 \theta$. Then the expression (32) is reduced to

$$
P_{\mathrm{suc}}(t)=\frac{1-\cos (2 \theta t+\theta)}{2}=\sin ^{2}[\theta(t+1 / 2)] .
$$

The latter is well known for the original Grover algorithm. It is seen from the formula for $\mathrm{L}^{t}$ that components of the Bloch vector are proportional to the factor $\eta^{t / 2}$, namely

$$
r_{x}(t) \propto \eta^{t / 2}, \quad r_{z}(t) \propto \eta^{t / 2} .
$$

Except for the value $\eta=1$, these components asymptotically tends to zero. Hence, the probability $P_{\text {suc }}(t)$ goes to $1 / 2$.

Let us proceed to the case (ii), when $\eta<A_{+}^{2}$. Due to $B^{2}=A_{+}^{2}-\eta$, the eigenvalues are expressed as

$$
\lambda_{ \pm}=A_{+} \pm B .
$$

For $\eta>0$, the eigenvalues are both strictly positive and $\lambda_{-}<\lambda_{+} \leq A_{+}+A_{-}=\cos 2 \theta<1$. So, the matrix $\mathrm{L}$ describes a contracting map. Due to $A_{+}^{2}-B^{2}=\eta$, we can write

$$
\frac{A_{+}}{\sqrt{\eta}}=\cosh \phi, \quad \frac{B}{\sqrt{\eta}}=\sinh \phi,
$$

where positive parameter $\phi$ reads as

$$
\phi=\frac{1}{2} \ln \left(\frac{A_{+}+B}{A_{+}-B}\right) .
$$

Hence, we can write $\lambda_{ \pm}=\sqrt{\eta} \exp ( \pm \phi)$. Similarly to the case (i), we diagonalize $L$ according to (A6). Now, the matrix of column eigenvectors and its inverse are represented as

$$
\begin{aligned}
\mathrm{X} & =\left(\begin{array}{cc}
\sin 2 \theta & \sin 2 \theta \\
A_{-}+B & A_{-}-B
\end{array}\right), \\
\mathrm{X}^{-1} & =\frac{1}{2 B \sin 2 \theta}\left(\begin{array}{lc}
B-A_{-} & \sin 2 \theta \\
B+A_{-} & -\sin 2 \theta
\end{array}\right) .
\end{aligned}
$$

Calculating the matrix $\mathrm{L}^{t}=X \mathrm{D}^{t} \mathrm{X}^{-1}$, we finally have

$$
\frac{\eta^{t / 2}}{B}\left(\begin{array}{cc}
B \cosh \phi t-A_{-} \sinh \phi t & \sinh \phi t \sin 2 \theta \\
-\eta \sinh \phi t \sin 2 \theta & B \cosh \phi t+A_{-} \sinh \phi t
\end{array}\right)
$$

since $A_{-}^{2}-B^{2}=\eta \sin ^{2} 2 \theta$. Due to $r_{x}(0)=\sin \theta$ and $r_{z}(0)=\cos \theta$, we finally get (33) and (34). The original Grover search is beyond the case (ii). Indeed, for $1 \leq$ $M \leq N / 2$ we have $\cos 2 \theta<1$, so that the condition $2 \sqrt{\eta}<(1+\eta) \cos 2 \theta$ is certainly violated with $\eta=1$.
[1] Galindo, A., Martin-Delgado, M.A.: Information and computation: classical and quantum aspects. Rev. Mod. Phys. 74, 347-423 (2002)

[2] Shor, P.W.: Polynomial-time algorithms for prime factorization and discrete logarithms on a quantum computer. SIAM J. Comput. 26, 1484-1509 (1997)

[3] Haase, D., Maier, H.: Quantum algorithms for number fields. Fortschr. Phys. 54, 866-881 (2006)

[4] Hallgren, S.: Polynomial-time quantum algorithms for Pell's equation and the principal ideal problem. J. ACM 54, 4 (2007)

[5] Childs, A.M., van Dam, W.: Quantum algorithms for algebraic problems. Rev. Mod. Phys. 82, 1-52 (2010)

[6] Grover, L.K.: Quantum mechanics helps in searching for a needle in a haystack. Phys. Rev. Lett. 79, 325-328 (1997)

[7] Grover, L.K.: Quantum computers can search arbitrarily large databases by a single query. Phys. Rev. Lett. 79, 4709-4712 (1997)

[8] Grover, L.K.: Quantum computers can search rapidly by using almost any transformation. Phys. Rev. Lett. 80, 4329-4332 (1998)

[9] Patel, A.D., Grover, L.K.: Quantum search. In: Kao, M.-Y. (ed.) Encyclopedia of Algorithms, pp. 1707-1716. Springer, New York (2016)

[10] Lomonaco, S.J., Kauffman, L.H.: Is Grover's algorithm a quantum hidden subgroup algorithm? Quantum Inf. Process. 6, 461-476 (2007)

[11] Bennett, C.H., Bernstein, E., Brassard, G., Vazirani, U.:
Strengths and weaknesses of quantum computing. SIAM J. Comput. 26, 1510-1523 (1997)

[12] Zalka, C.: Grover's quantum searching algorithm is optimal. Phys. Rev. A 60, 2746-2751 (1999)

[13] Biham, E., Biham, O., Biron, D., Grassl, M., Lidar, D.A.: Grover's quantum search algorithm for an arbitrary initial amplitude distribution. Phys. Rev. A 60, 2742-2745 (1999)

[14] Galindo, A., Martin-Delgado, M.A.: Family of Grover's quantum-searching algorithms. Phys. Rev. A 62, 062303 (2000)

[15] Biham, E., Biham, O., Biron, D., Grassl, M., Lidar, D.A., Shapira, D.: Analysis of generalized Grover quantum search algorithms using recursion equations. Phys. Rev. A 63, 012310 (2000)

[16] Biham, E., Kenigsberg, D.: Grover's quantum search algorithm for an arbitrary initial mixed state. Phys. Rev. A 66, 062301 (2002)

[17] Watrous J.: The Theory of Quantum Information. Cambridge University Press, Cambridge (2018)

[18] Deutsch, D.: Quantum theory, the Church-Turing principle and the universal quantum computer. Proc. R. Soc. Lond. A 400, 97-117 (1985)

[19] Nielsen, M.A., Chuang, I.L.: Quantum Computation and Quantum Information. Cambridge University Press, Cambridge (2000)

[20] Braunstein, S.L., Pati, A.K.: Speed-up and entanglement in quantum searching. Quantum Inf. Comput. 2, 399-409 (2002) 
[21] Jozsa, R., Linden, N.: On the role of entanglement in quantum-computational speed-up. Proc. R. Soc. Lond. A 459, 2011-2032 (2003)

[22] Baumgratz, T., Cramer, M., Plenio, M.B.: Quantifying coherence. Phys. Rev. Lett. 113, 140401 (2014)

[23] Streltsov, A., Adesso, G., Plenio, M.B.: Quantum coherence as a resource. Rev. Mod. Phys. 89, 041003 (2017)

[24] Adesso, G., Bromley, T.R., Cianciaruso, M.: Measures and applications of quantum correlations. J. Phys. A: Math. Theor. 49, 473001 (2016)

[25] Hu, M.-L., Fan, H.: Relative quantum coherence, incompatibility, and quantum correlations of states. Phys. Rev. A 95, 052106 (2017)

[26] Hu, M.-L., Hu, X., Wang, J.-C., Peng, Y., Zhang, Y.-R., Fan, H.: Quantum coherence and quantum correlations. E-print arXiv:1703.01852 [quant-ph] (2017)

[27] Vedral, V.: The role of relative entropy in quantum information theory. Rev. Mod. Phys. 74, 197-234 (2002)

[28] Rastegin, A.E.: Quantum coherence quantifiers based on the Tsallis relative $\alpha$ entropies. Phys. Rev. A 93, 032136 (2016)

[29] Chitambar, E., Gour, G.: Comparison of incoherent operations and measures of coherence. Phys. Rev. A 94, 052336 (2016)

[30] Shao, L.-H., Li, Y., Luo, Y., Xi, Z.: Quantum coherence quantifiers based on the Rényi $\alpha$-relative entropy. Commun. Theor. Phys. 67, 631-636 (2017)

[31] Streltsov, A., Kampermann, H., Wölk, S., Gessner, M., Bruß, D.: Maximal coherence and the resource theory of purity. New J. Phys. 20, 053058 (2018)

[32] Shao, L.-H., Xi, Z., Fan, H., Li, Y.: Fidelity and tracenorm distances for quantifying coherence. Phys. Rev. A 91, 042120 (2015)

[33] Rana, S., Parashar, P., Lewenstein, M.: Trace-distance measure of coherence. Phys. Rev. A 93, 012110 (2016)

[34] Zhang, H.-J., Chen, B., Li, M., Fei, S.-M., Long, G.-L.: Estimation on geometric measure of quantum coherence. Commun. Theor. Phys. 67, 166-170 (2017)

[35] Cheng, S., Hall, M.J.W.: Complementarity relations for quantum coherence. Phys. Rev. A 92, 042101 (2015)

[36] Singh, U., Pati, A.K., Bera, M.N.: Uncertainty relations for quantum coherence. Mathematics 4, 47 (2016)

[37] Peng, Y., Zhang, Y.-R., Fan, Z.-Y., Liu, S., Fan, H.: Complementary relation of quantum coherence and quantum correlations in multiple measurements. E-print arXiv:1608.07950 [quant-ph] (2016)

[38] Yuan, X., Bai, G., Peng, T., Ma, X.: Quantum uncertainty relation using coherence. Phys. Rev. A 96, 032313 (2017)

[39] Rastegin, A.E.: Uncertainty relations for quantum coherence with respect to mutually unbiased bases. Front. Phys. 13, 130304 (2018)

[40] Bera, M.N., Qureshi, T., Siddiqui, M.A., Pati, A.K.: Duality of quantum coherence and path distinguishability. Phys. Rev. A 92, 012118 (2015)

[41] Bagan, E., Bergou, J.A., Cottrell, S.S., Hillery, M.: Relations between coherence and path information. Phys. Rev. Lett. 116, 160406 (2016)

[42] Qureshi, T., Siddiqui, M.A.: Wave-particle duality in $N$ path interference. Ann. Phys. 385, 598-604 (2017)

[43] Hillery, M.: Coherence as a resource in decision problems: The Deutsch-Jozsa algorithm and a variation. Phys. Rev. A 93, 012111 (2016)

[44] Shi, H.-L., Liu, S.-Y., Wang, X.-H., Yang, W.-L., Yang, Z.-Y., Fan, H.: Coherence depletion in the Grover quantum search algorithm. Phys. Rev. A 95, 032307 (2017)

[45] Anand, N., Pati, A.K.: Coherence and entanglement monogamy in the discrete analogue of analog Grover search. E-print arXiv:1611.04542 [quant-ph] (2016)

[46] Rastegin, A.E.: On the role of dealing with quantum coherence in amplitude amplification. Quantum Inf. Process. 17, 179 (2018)

[47] Reitzner, D., Hillery, M.: Grover search under localized dephasing. E-print arXiv:1712.06558 [quant-ph] (2017)

[48] Hillery, M., Bergou, J., Feldman, E.: Quantum walks based on an interferometric analogy, Phys. Rev. A 68, 032314 (2003) 